\title{
SLCO1B1 *15 haplotype is associated with rifampin-induced liver injury
}

\author{
LING-MIN LI $^{*}{ }^{*}$, LEI CHEN $^{1 *}$, GUO-HONG DENG ${ }^{2}$, WEN-TING TAN ${ }^{2}$, \\ YUN-JIE DAN $^{2}$, RONG-QUAN WANG ${ }^{1}$ and WEN-SHENG CHEN ${ }^{1}$ \\ Institutes of ${ }^{1}$ Digestive Disease, and ${ }^{2}$ Infectious Diseases, Southwest Hospital, \\ The Third Military Medical University, Chongqing 400038, P.R. China
}

Received January 10, 2012 ; Accepted April 20, 2012

DOI: $10.3892 / \mathrm{mmr} .2012 .900$

\begin{abstract}
The organic anion transporting polypeptide 1B1 (OATP1B1, encoded by $S L C O 1 B 1$ ) plays an important role in the transport of endogenous and xenobiotic compounds, such as bile acids and rifampin. In this study, the association between OATP1B1 polymorphisms and rifampin hepatotoxicity was investigated using integrated population genetic analysis and functional studies. A total of 273 unrelated patients treated with rifampin were recruited. The allele frequencies were examined in patients with drug (rifampin)-induced liver injury (DILI) $(n=118)$ and without (non-DILI) $(n=155)$. Functional analyses were conducted to determine whether the inhibition of bile acids by rifampin was associated with OATP1B1 variants. In the present study, 24 single nucleotide polymorphisms (SNPs) in OATP1B1 were detected in a Chinese population, with two of them causing an amino acid change (rs2306283 and rs4149056). The haplotypes constructed by these two SNPs were OATP1B1 *1a, *1b, ${ }^{*} 5$ and ${ }^{*} 15$, with their respective frequencies being $23.44,66.30,0.73$ and $9.52 \%$ in a total of 273 individuals. The logistic regression analysis indicated that the *15 haplotype was associated with susceptibility to DILI $(\mathrm{p}=0.03, \mathrm{OR}=2.04,95 \% \mathrm{CI} 1.05-3.96)$. The frequency of the *15 haplotype in DILI patients was significantly higher than that in non-DILI patients $(\mathrm{p}=0.03)$. In the subgroup analysis, the *15 haplotype was associated with susceptibility to cholestatic/ mixed injury ( $\mathrm{p}=0.03, \mathrm{OR}=2.31,95 \% \mathrm{CI} 1.06-5.02)$. Functional assessment of the OATP1B1 *15 haplotype revealed that the activity of bile acid uptake was markedly reduced compared to the three other haplotypes. In the inhibition study, the inhibi-
\end{abstract}

Correspondence to: Dr Wen-Sheng Chen, Institute of Digestive Disease, Southwest Hospital, The Third Military Medical University, Chongqing 400038, P.R. China

E-mail: wensheng-chen1@hotmail.com

*Contributed equally

Key words: organic anion transporting polypeptide 1B1, polymorphism, haplotype, pharmacogenetics, rifampin-induced liver injury tion by rifampin in the * 15 haplotype was greater compared to that in the other haplotypes. These results suggest that the OATP1B1 *15 haplotype is an important predisposing factor for rifampin-induced liver injury.

\section{Introduction}

Rifampin (RMP) is one of the major antituberculosis drugs, a second-choice antistaphylococcal agent and an effective medicine in controlling pruritus in primary biliary cirrhosis (1-3). The common adverse reactions to RMP include fever, nausea, vomiting, diarrhea, abdominal pain, hemolytic anemia, thrombocytopenia, liver injury and tubular defects. The hepatotoxicity induced by RMP was first reported in 1971, and it has an incidence rate of 5 to $12.5 \%(4,5)$. RMP-induced hepatotoxicity can be divided into three categories: hepatocellular, cholestatic or mixed-type injury (6). In spite of the well-accepted toxicity of RMP in vivo, its toxic mechanism remains unclear. Recently, one important mechanism was determined to be a rise in serum bilirubin via competitive inhibition of hepatocyte bilirubin transport by RMP (7). The inhibition of the hepatic uptake of bile acid has been proposed as the mechanism responsible for RMP-induced cholestasis (8). Notably, the genetic polymorphisms of bile acid transporters have been reported to be closely related to the susceptibility to RMP hepatotoxicity (9).

Organic anion transporting polypeptide 1B1 (OATP1B1, encoded by $S L C O 1 B 1$ ) is one of the most important bile acid transporters. It is mainly expressed on the basolateral membrane of human hepatocytes and is responsible for bilirubin uptake $(10,11)$. Several studies have demonstrated that OATP1B1 plays an important role in the hepatic uptake of RMP (12). In particular, it has displayed a great capability for RMP transport in HeLa cells, with RMP uptake being markedly decreased by the OATP1B1 allelic variants (13). To date, however, many studies of RMP hepatotoxicity have concentrated on the transporters that export RMP, and few studies have been published concerning the importance of the transporters that uptake RMP. In fact, RMP inhibits the uptake of bile acids by OATP1B1 (14). For example, RMP inhibits OATP1B1-mediated substrate uptake into the liver in a Xenopus laevis oocyte expression system (13). Furthermore, variants of OATP1B1 in different populations influence the 
uptake of bilirubin and RMP, thus directly leading to changes in the susceptibility to RMP hepatotoxicity (15). The association between functional single nucleotide polymorphisms (SNPs) in the OATP1B1 gene and RMP-induced liver injury in a Chinese population, remains, however, unclear.

The naturally occurring sequence variations in the $S L C O 1 B 1$ gene leads to genetic differences that affect susceptibility to RMP hepatotoxicity. Thus, in the present study, SNPs in SLCOIB1 were systemically screened, and the association of the $S L C O 1 B 1$ polymorphisms with susceptibility to RMP-induced liver injury in a Chinese population was investigated. Then, cells expressing the variants of OATP1B1 were used as an in vitro model to assess the inhibitory effects of RMP on bile acid transport.

\section{Materials and methods}

Participants. A total of 273 unrelated patients treated with RMP were recruited in this study. All of the participants lived in Chongqing, China. The patients were admitted to the Institute of Digestive Disease and Infectious Disease of the Southwest Hospital and the Chongqing Antituberculosis Clinic between January 1, 2009 and January 1, 2011. They were enrolled in the study if they met the following criteria: i) were $18-65$ years of age, and ii) had RMP treatment prescribed for $>3$ months. Patients were not eligible if they had pre-existing cardiovascular, renal, hepatic, hematologic or immunologic diseases. Drug-induced liver injury (DILI) was defined as serum alanine aminotransferase (ALT) levels three or more times the upper limit of normal (ULN), and/or serum bilirubin levels two or more times the ULN. Patients diagnosed with DILI had liver injury occurring after RMP retreatment and had previously normal serum ALT levels. The type of liver damage was classified according to the International Consensus Meeting Criteria $(16,17)$ using ALT and alkaline phosphatase activity, expressed as a multiple of the upper limit of normality, to determine the ratio (R) of ALT/AP. The type of liver damage was defined as hepatocellular when $R \geq 5$, cholestatic when $\mathrm{R} \leq 2$ and mixed when $2<\mathrm{R}<5$. The protocol for this study was approved by the Ethics Committee of the Southwest Hospital, Chongqing, China. Informed consent was obtained from the patients, and patient confidentiality was preserved according to the guidelines for studies of human subjects.

Discovery of SNPs and determination of haplotypes. SNP screening of the $\sim 9.1-\mathrm{kb}$ region of SLCO1B1 (NT_009714 http://www.ncbi.nlm.nih.gov/gene/10599) continuously from the 5 ' flanking to 3 ' flanking genomic regions (including all exons, relevant exon-intron boundaries and $600 \mathrm{bp}$ of the promoter region) was performed by polymerase chain reaction (PCR) direct sequencing. SNP candidates were identified by the PolyPhred program and inspected by two observers. SNP positions and individual genotypes were confirmed by reamplifying and resequencing the SNP site from the opposite strand. The screening panel included 32 unrelated individuals randomly selected from the RMP-induced liver injury group. The sample size provided a $95 \%$ probability of detecting haplotypes with a minimum frequency of $5.4 \%$ (18). The pairwise linkage disequilibrium (LD) measures were calculated using the Arlequin package (University of Geneva, Geneva,
Switzerland), and the value of $r^{2}$ was plotted using the LDA program.

SLCO1B1 genotyping. Genomic DNA was extracted from peripheral blood leukocytes using $2 \mathrm{ml}$ of whole blood and the EZNA blood DNA kit (Omega Bio-Tek). The participants in the study were genotyped for the 388A $>$ G (N130D, rs2306283) and 521T $>$ C (V174A, rs4149056) SNPs in the SLCO1B1 gene by allelic discrimination with TaqMan ${ }^{\circledR}$ using Minor Groove Binder (MGB) DNA oligonucleotide technology. Genotyping for the 388A $>$ G and 521T>C SNPs was performed with validated 6-carboxyfluorescein (FAM) or VIC dye-labeled MGB-TaqMan Genotyping Assays (Applied Biosystems, Foster City, CA, USA). The accuracy of the SNP genotyping data was validated by direct sequencing of $8 \%$ masked, random samples of patients.

Construction of OATP1B1 variants and functional analysis. The pcDNA3.1 OATP1B1 *1a (388A521T), "1b (388G521T), *5 (388A521C) and *15 (388G521C) vectors were kindly provided by Dr Yoshio Kameyama (Chiba University, Japan). These full-lengths of OATP1B1 were released by Kpnl and Notl double digestion at $37^{\circ} \mathrm{C}$ for $2 \mathrm{~h}$. These fragments were cloned into the Kpnl and Notl sites of pcDNA3.1/ CT-GFP-TOPO (Invitrogen, Carlsbad, CA, USA) mock vectors. The resulting vectors were then sequenced. HEK293 cells grown in a 12-well plate were transfected with variants of OATP1B1 with Lipofectamine 2000 (Invitrogen) and selected with 1,200 $\mu \mathrm{g} / \mathrm{ml}$ zeocin (Gibco, Grand Island, NY, USA). Those colonies stably expressing OATP1B1 were cultured in $10 \%$ fetal bovine serum with zeocin $(800 \mu \mathrm{g} /$ $\mathrm{ml}$ ). The pcDNA3.1-GFP vector alone was used to obtain background activity (mock). The expression of the OATP1B1 variants was verified by reverse transcriptase (RT)-PCR and double-fluorescence confocal microscopy using the protocol described by Kameyama et al (19). Transport studies of RMP and $\left({ }^{3} \mathrm{H}\right)$ taurocholic acid (TCA) were carried out as previously described (14,20). HEK293 cells stably expressing OATP1B1 were grown on poly-D-lysine-coated 12-well culture plates. The expression of OATP1B1 was induced by $10 \mathrm{mM}$ sodium butyrate for $24 \mathrm{~h}$. The HEK293 cells with the highest transport activities were used in the functional analysis. Then, experiments were initiated by gently removing medium and adding $200 \mu \mathrm{l}$ transport medium (118 mM NaCl, $23.8 \mathrm{mM}$ $\mathrm{NaHCO}_{3}, 4.83 \mathrm{mM} \mathrm{KCl}, 0.96 \mathrm{mM} \mathrm{KH}_{2} \mathrm{PO}_{4}, 1.20 \mathrm{mM} \mathrm{MgSO}_{4}$, $12.5 \mathrm{mM}$ HEPES, $5 \mathrm{mM}$ glucose and $1.53 \mathrm{mM} \mathrm{CaCl}_{2}$ adjusted to $\mathrm{pH}$ 7.4) containing radiolabeled drug $\left({ }^{3} \mathrm{H}\right) \mathrm{TCA}(1 \mu \mathrm{M})$ (PerkinElmer Life Sciences, Boston, MA, USA) for $10 \mathrm{~min}$. RMP (Sigma, USA) was first dissolved in dimethylsulfoxide (DMSO; 100\%) and subsequently diluted 1:100 in the incubation medium. Transport for RMP was determined after $30 \mathrm{~min}$ of incubation with $0,10,20,50,75$ and $100 \mu \mathrm{M}$ at $37^{\circ} \mathrm{C}$. At the end of the experiment, cells were washed with ice-cold PBS three times and lysed with $350 \mu 1$ of $0.1 \mathrm{~N} \mathrm{NaOH}$. Then, $10 \mu \mathrm{l}$ aliquots of cell lysate were used to determine protein concentration by the bicinchoninic acid (BCA) assay method. Retained cellular radioactivity was quantified by liquid scintillation spectrometry (Beckman LS 6500 Multi-Purpose Scintillation Counter). The OATP1B1-mediated uptake was calculated after subtracting the uptake by the background 
from the uptake by OATP1B1 variant-transfected cells at each concentration.

Statistical analysis. Statistical analysis was performed using SPSS software (Version 11.5; SPSS Inc., Chicago, IL, USA). Genotype and haplotype frequencies were determined by gene counting, and the significance of deviations from Hardy-Weinberg equilibrium was tested using the random permutation procedure implemented in the Arlequin package. The association between genotyped polymorphisms and disease risk was estimated with an unconditional logistic regression analysis and represented as p-values, odds ratios (ORs) and 95\% confidence intervals (95\% CIs). To estimate the kinetic parameters for the uptake of $\left({ }^{3} \mathrm{H}\right) \mathrm{TCA}$ by the HEK293-OATP1B1-overexpressing cell system, $\left({ }^{3} \mathrm{H}\right) \mathrm{TCA}$ uptake rates were calculated by subtracting the transport rate of background cells from that of OATP1B1-expressing cells. The transport kinetics data were analyzed with the Prism software (GraphPad Software, Inc., La Jolla, CA, USA). The kinetic parameter was defined as pmol $\mathrm{x} \mathrm{mg} \mathrm{protein}{ }^{-1} \mathrm{x} \mathrm{min}^{-1}$. $\mathrm{p}<0.05$ was considered a significant difference for all statistical analyses.

\section{Results}

Sequence variations in the SLCO1B1 genomic region. Sequencing of the 9,126-bp SLCO1B1 genomic regions in the 32 samples revealed 24 SNPs (Table I). Of these variations, 4 were located in coding exons ( 2 synonymous and 2 nonsynonymous), 14 in introns and 4 in the 3 ' untranslated region. This study focused on the non-synonymous variants, snp6 (388A > G, rs2306283) and snp11 (521T>C, rs4149056), as they resulted in amino acid changes. Analysis of the genotyping data generated by HapMap revealed that SLCO1B1 had three haplotype blocks. Snp6 and snp11 were in the third block and had low paired $\operatorname{LD}\left(\mathrm{r}^{2}=0.036\right)$.

Case-control study. During 2009-2011, 273 patients were enrolled in this study, of whom 118 fulfilled the DILI criteria. There were no significant differences between DILI and non-DILI group populations in terms of age (mean 40.5 vs. 39.3 years) and gender ratio (female 51.7 vs. $45.1 \%$ ). The DILI patients were classified into two subgroups according to the International Consensus Meeting Criteria: 58 patients with hepatocellular injury (HC group) and 60 patients with cholestatic/mixed injury (CS/mixed group). There were no significant differences between the two groups in terms of age, gender, drinking history and smoking status (Table II).

All of the recruited patients were successfully genotyped for the 388A $>\mathrm{G}$ (rs2306283) and 521T $>C$ (rs4149056) SNPs. The genotype distributions of the $388 \mathrm{~A}>\mathrm{G}$ and $521 \mathrm{~T}>\mathrm{C}$ frequencies in the HC, CS/mixed and non-DILI groups are given in Table III. The genotype distributions of these two SNPs $(388 \mathrm{~A}>\mathrm{G}, 521 \mathrm{~T}>\mathrm{C})$ were in Hardy-Weinberg equilibrium in each group (for non-DILI group, $\mathrm{p}=0.1012,0.6367$; for HC group, $\mathrm{p}=0.4121,0.2958$; for $\mathrm{CS} /$ mixed group, $\mathrm{p}=0.1650$, 0.3806). The univariate analysis of the factors analyzed and their association with the risk of DILI are also given in Table III. The frequency of the $388 \mathrm{~A}>\mathrm{G}$ polymorphism was not significantly different between the non-DILI group and
Table I. Positions and frequencies of SNPs within the human SLCO1B1 gene.

\begin{tabular}{|c|c|c|c|c|}
\hline No. & $\mathrm{SNP}^{\mathrm{a}}$ & db SNP ID & Frequency $^{c}$ & Region \\
\hline 1 & $\mathrm{~T} 11225 \mathrm{C}$ & rs 74474044 & 0.046 & Intron 2 \\
\hline 2 & G11250A & rs4149021 & 0.171 & Intron 2 \\
\hline 3 & T42279G & rs2291073 & 0.258 & Intron 3 \\
\hline 4 & A44132C & rs61628014 & 0.015 & intron 4 \\
\hline 5 & C44205A & rs4149036 & 0.437 & Intron 4 \\
\hline 6 & A46203G (Asn/Asp) & rs2306283 & 0.234 & Exon 5 \\
\hline 7 & A46461T & rs4149044 & 0.437 & Intron 5 \\
\hline 8 & G46485A & rs4149045 & 0.453 & Intron 5 \\
\hline 9 & G46487A & rs4149046 & 0.218 & Intron 5 \\
\hline 10 & TACTTG47863_Del & rs4149096 & 0.451 & Intron 5 \\
\hline 11 & T48014C (Ala/Val) & rs4149056 & 0.193 & Exon 6 \\
\hline 12 & T48064C (Leu/Leu) & rs4149057 & 0.290 & Exon 6 \\
\hline 13 & C48090T (Phe/Phe) & rs2291075 & 0.419 & Exon 6 \\
\hline 14 & G48274A & rs12580064 & 0.096 & Intron 6 \\
\hline 15 & $\mathrm{C} 48452 \mathrm{~T}$ & rs2291076 & 0.267 & Intron 7 \\
\hline 16 & T48686A & rs2291077 & 0.250 & Intron 7 \\
\hline 17 & CTT68526_Del & rs4149098 & 0.225 & Intron 10 \\
\hline 18 & TTT68582_Del & - & 0.016 & Intron 10 \\
\hline 19 & G91303T & rs987839 & 0.016 & Intron 12 \\
\hline 20 & A91359G & - & 0.440 & Intron 14 \\
\hline 21 & C108670T & rs 72655363 & 0.031 & 3'-UTR \\
\hline 22 & $\mathrm{~T} 108755 \mathrm{C}$ & rs4149085 & 0.375 & 3'-UTR \\
\hline 23 & $\mathrm{~T} 109027 \mathrm{G}$ & rs4149087 & 0.218 & 3'-UTR \\
\hline 24 & A109051G & rs4149088 & 0.218 & 3'-UTR \\
\hline
\end{tabular}

Del, one-base deletion; UTR, untranslated region. ${ }^{\text {aThe position of }}$ the SNPs is related to the first nucleotide of the open reading frame of the $S L C O 1 B 1$ gene; the allele on the right is the minor allele. ${ }^{b} \mathrm{db}$ gene ID: NT_009714 in http://www.ncbi.nlm.nih.gov/SNP. ${ }^{c}$ Frequencies of minor alleles. Allele frequencies for each SNP were determined by gene counting on the 32 unrelated samples (12 chromosomes).

the other two groups $(p=0.5562,0.5136)$. For the 521T $>C$ polymorphism, however, there was a significant difference in the haplotype frequencies between the DILI and non-DILI patients. For this SNP, the frequency of the C allele was 0.1833 in the HC group, 0.1207 in the CS/mixed group and 0.0645 in the non-DILI group. Subjects bearing the 521C allele had an increased susceptibility to RMP-induced liver injury compared to those with the 521T allele $(\mathrm{p}=0.0233)$.

Next, the frequencies of the SLCO1B1 haplotypes containing the $388 \mathrm{~A}>\mathrm{G}$ and $521 \mathrm{~T}>\mathrm{C}$ SNPs (Table IV) were analyzed to determine which haplotype specifically correlated with RMP-induced liver injury. Four haplotypes were observed, with the *5 (388A521C) haplotype having a frequency $<5 \%$. The frequencies of the four haplotypes (" $1 \mathrm{a}$, * $1 b,{ }^{*} 5$ and ${ }^{*} 15$ ) in the study population were $23.44,66.30,0.73$ and $9.52 \%$, respectively. The haplotype distributions for the RMP-induced liver injury and non-DILI groups are shown in Table IV. The overall haplotype frequency was significantly different between the CS/mixed injury and non-DILI groups $\left(\chi^{2}=14.6551\right.$, global $\left.\mathrm{p}=0.0221\right)$. The respective genotype 
Table II. Clinical and laboratory data of patients in the non-DILI and DILI groups and subgroups.

\begin{tabular}{|c|c|c|c|c|c|c|c|}
\hline \multirow[t]{2}{*}{ Characteristics } & \multirow{2}{*}{$\begin{array}{l}\text { Non-DILI } \\
(\mathrm{n}=155)\end{array}$} & \multicolumn{2}{|c|}{ DILI } & \multicolumn{2}{|c|}{ CS/mixed injury, (n) } & \multicolumn{2}{|c|}{ HC injury, (n) } \\
\hline & & $(\mathrm{n}=118 ; \%)$ & p-value ${ }^{a}$ & $(\mathrm{n}=60 ; \%)$ & p-value ${ }^{a}$ & $(\mathrm{n}=58 ; \%)$ & p-value ${ }^{a}$ \\
\hline Age (years) & $39.36 \pm 15.54$ & $40.53 \pm 13.45$ & 0.052 & $39.03 \pm 13.45$ & 0.084 & $41.16 \pm 14.65$ & 0.046 \\
\hline \multicolumn{8}{|l|}{ Gender } \\
\hline Male, n (\%) & $80(54.83)$ & $57(48.30)$ & 0.293 & $26(43.33)$ & 0.045 & $31(53.44)$ & 0.432 \\
\hline Female, n (\%) & $75(45.17)$ & $61(51.70)$ & & $34(56.67)$ & & $27(46.56)$ & \\
\hline \multicolumn{8}{|l|}{ Smoking status } \\
\hline Present, n (\%) & $85(54.83)$ & $45(38.13)$ & 0.070 & $21(35)$ & 0.092 & $24(41.37)$ & 0.245 \\
\hline Absent, n (\%) & $70(45.17)$ & $73(61.87)$ & & $39(65)$ & & $34(58.63)$ & \\
\hline \multicolumn{8}{|l|}{ Drinking history } \\
\hline Present, n (\%) & $95(61.29)$ & $21(17.78)$ & 0.227 & $9(15.51)$ & 0.228 & $12(20.68)$ & 0.170 \\
\hline Absent, n (\%) & $60(38.71)$ & $97(82.22)$ & & $51(84.49)$ & & $46(79.32)$ & \\
\hline \multicolumn{8}{|l|}{ Duration of treatment } \\
\hline $\begin{array}{l}\text { Total bilirubin } \\
\text { (normal, }<21 \mu \mathrm{mol} / \mathrm{l} \text { ) }\end{array}$ & - & $90.1 \pm 82.1$ & & $139.9 \pm 118.5$ & & $41.8 \pm 38.1$ & \\
\hline $\begin{array}{l}\text { Direct bilirubin } \\
\text { (normal, }<21 \mu \mathrm{mol} / \mathrm{l})\end{array}$ & - & $55.8 \pm 22.6$ & & $87.7 \pm 38.4$ & & $22.7 \pm 17.4$ & \\
\hline $\begin{array}{l}\mathrm{AST}^{\mathrm{b}} \\
(\text { normal, }<42 \mathrm{IU} / \mathrm{l})\end{array}$ & - & $324.6 \pm 244.2$ & & $303.7 \pm 254.4$ & & $337.0 \pm 208.5$ & \\
\hline $\begin{array}{l}\mathrm{ALT}^{\mathrm{b}} \\
\text { (normal, <42 IU/1) }\end{array}$ & - & $370.4 \pm 309.1$ & & $338.0 \pm 290.4$ & & $403.4 \pm 329.5$ & \\
\hline $\begin{array}{l}\mathrm{GGT}^{\mathrm{b}} \\
\text { (normal, <45 IU/1) }\end{array}$ & - & $232.4 \pm 122.6$ & & $287.2 \pm 183.2$ & & $178.2 \pm 100.5$ & \\
\hline $\begin{array}{l}\text { Total bile acid } \\
(\text { normal },<10 \mu \mathrm{mol} / \mathrm{l}) 1\end{array}$ & - & $11.2 \pm 4.8$ & & $13.2 \pm 5.54$ & & $10.4 \pm 3.2$ & \\
\hline
\end{tabular}

${ }^{\mathrm{a}} \mathrm{p}$-values were for the comparison with the non-DILI (drug-induced liver injury) group. For age and duration of treatment, p-values were given by paired-t test; for gender, smoking status and drinking history, p-values were given by the $\chi^{2}$-test. ${ }^{b}$ The laboratory parameters are all peak values before RMP treatment.

Table III. OATP1B1 genotype distributions in participants.

\begin{tabular}{|c|c|c|c|c|c|}
\hline Variant & Genotype & Non-DILI, n (\%) & DILI, n (\%) & $\mathrm{CS} /$ mixed injury, n (\%) & HC injury, n (\%) \\
\hline c. $388 \mathrm{~A}>\mathrm{G}$ & $\mathrm{A} / \mathrm{A}$ & $12(7.74)$ & $11(9.32)$ & $7(11.67)$ & $4(6.90)$ \\
\hline \multirow[t]{5}{*}{ (p.N130D) } & $\mathrm{A} / \mathrm{G}$ & $48(30.97)$ & $38(32.20)$ & $20(33.33)$ & $18(31.03)$ \\
\hline & $\mathrm{G} / \mathrm{G}$ & $95(61.29)$ & $69(58.47)$ & $33(55.00)$ & $36(62.07)$ \\
\hline & A allele & $72(23.23)$ & $60(25.42)$ & $34(28.33)$ & $26(22.41)$ \\
\hline & G allele & $238(76.77)$ & $176(74.57)$ & $86(71.67)$ & $90(77.59)$ \\
\hline & p-value ${ }^{a}$ & & 0.5562 & 0.5136 & 0.6020 \\
\hline c. $521 \mathrm{~T}>\mathrm{C}$ & $\mathrm{T} / \mathrm{T}$ & $136(87.74)$ & $83(70.33)$ & $39(65.00)$ & $44(75.86)$ \\
\hline \multirow{5}{*}{ (p.V174A) } & $\mathrm{T} / \mathrm{C}$ & $18(11.61)$ & $34(28.81)$ & $20(33.30)$ & $14(24.14)$ \\
\hline & $\mathrm{C} / \mathrm{C}$ & $1(0.65)$ & $1(0.84)$ & $1(1.67)$ & 0 \\
\hline & $\mathrm{T}$ allele & $290(93.55)$ & $200(84.74)$ & $98(81.67)$ & $102(87.93)$ \\
\hline & $\mathrm{C}$ allele & $20(6.45)$ & $36(15.25)$ & $22(18.33)$ & $14(12.07)$ \\
\hline & $\mathrm{p}$-value ${ }^{\mathrm{a}}$ & & 0.0233 & 0.0336 & 0.0146 \\
\hline
\end{tabular}

${ }^{\mathrm{a} p}$-value was compared with the non-DILI (drug-induced liver injury) group.

frequencies for ${ }^{*} 1 \mathrm{a},{ }^{*} 1 \mathrm{~b},{ }^{*} 5$ and ${ }^{*} 15$ were $23.72,61.02,1.69$ and $13.56 \%$ in DILI patients, and 23.22, 70.32, 0 and 6.45\% in nonDILI patients. The common risk haplotype, ${ }^{*} 15$ (388G521C), was significantly higher in the CS/mixed group (15.0\%) than in the non-DILI group $(6.45 \%)$. The haplotype *1b (388G521T) was higher in the non-DILI $(70.32 \%)$ than in the HC $(65.51 \%)$ 
Table IV. Results of the association test for the OATP1B1 haplotypes between the non-DILI and DILI groups.

\begin{tabular}{|c|c|c|c|c|c|c|c|c|c|c|}
\hline \multirow[t]{2}{*}{ Characteristics } & \multirow{2}{*}{$\begin{array}{l}\text { Non-DILI } \\
(\mathrm{n}=310)\end{array}$} & \multicolumn{3}{|c|}{ DILI (n=236) } & \multicolumn{3}{|c|}{ CS/mixed injury $(n=120)$} & \multicolumn{3}{|c|}{ HC injury $(n=116)$} \\
\hline & & $\mathrm{n}(\%)$ & p-value ${ }^{a}$ & $\begin{array}{c}\text { OR } \\
(95 \% \mathrm{CI})\end{array}$ & $\mathrm{n}(\%)$ & p-value ${ }^{a}$ & $\begin{array}{c}\text { OR } \\
(95 \% \mathrm{CI})\end{array}$ & $\mathrm{n}(\%)$ & p-value ${ }^{a}$ & $\begin{array}{c}\text { OR } \\
(95 \% \mathrm{CI})\end{array}$ \\
\hline
\end{tabular}

$\begin{array}{lccc}\text { Haplotype } & & & \\ { }^{*} 1 \mathrm{a}(388 \mathrm{~A} 521 \mathrm{~T}) & 72(23.22) & 56(23.73) & 0.080 \\ { }^{*} 1 \mathrm{~b}(388 \mathrm{G} 521 \mathrm{~T}) & 218(70.32) & 144(61.02) & 0.080 \\ { }^{*} 5(388 \mathrm{~A} 521 \mathrm{C}) & 0(0) & 4(1.69) & 0.007 \\ { }^{*} 15(388 \mathrm{G} 521 \mathrm{C}) & 20(6.45) & 32(13.56) & \mathbf{0 . 0 3 0}\end{array}$

$\begin{array}{cc}30(25.00) & 0.19 \\ 68(56.67) & 0.06 \\ 4(3.33) & 0.01 \\ 18(15.00) & \mathbf{0 . 0 2}\end{array}$

$\begin{array}{cc}26(22.41) & 0.82 \\ 76(65.51) & 0.07 \\ 0(0) & - \\ 14(11.67) & 0.02\end{array}$

Haplotype groups ${ }^{\mathrm{b}}$

$-/ \mathrm{c}^{\mathrm{c}}$

${ }^{*} 15 / \mathrm{-}^{\mathrm{d}}$
$137(86.46) 93(78.81)$

$18(11.61) \quad 25(21.18)$
0.030

$\begin{array}{cr} & 42(75.00) \\ \mathbf{2 . 0 4} & 14(25.00) \\ (\mathbf{1 . 0 5 - 3 . 9 6 )} & \end{array}$

\subsection{3}

.06

${ }^{\mathrm{a}}$ Odds ratios (OR) and 95\% confidence intervals (CIs) were calculated by comparison to the non-DILI group. ${ }^{\mathrm{b}} \mathrm{p}$-value was calculated using logistic regression analysis. ${ }^{\mathrm{c}}-/$ - including $\left({ }^{*} 1 \mathrm{a} /{ }^{*} 1 \mathrm{a},{ }^{*} 1 \mathrm{a} /{ }^{*} 1 \mathrm{~b},{ }^{*} 1 \mathrm{~b} /{ }^{*} 1 \mathrm{~b}\right)$. Significant $\mathrm{p}$-values $(<0.05)$ are in boldface. ${ }^{\mathrm{d} *} 15 /-$ indicates at least one "15 allele $\left(" 1 \mathrm{~b} /{ }^{*} 15,{ }^{*} 1 \mathrm{a} /{ }^{*} 15,{ }^{*} 15 / * 15\right)$.

A

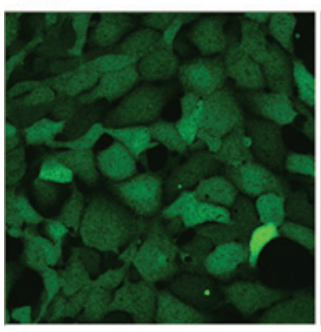

mock

B

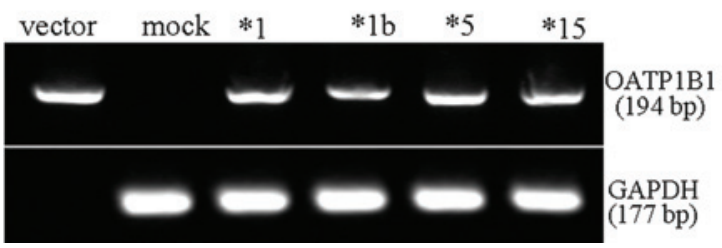

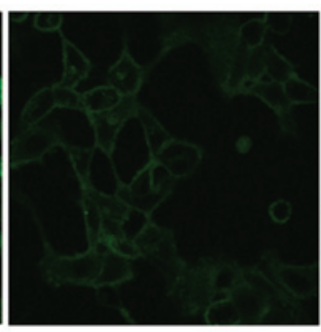

OATP1B1*1a

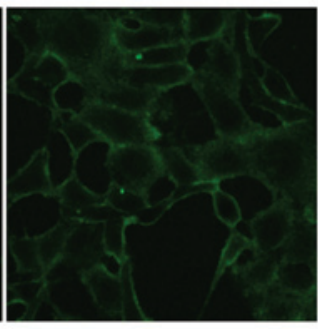

OATP1B $1 * 1 \mathrm{~b}$

C

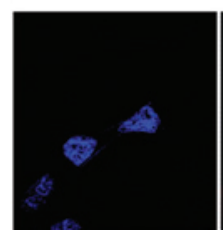

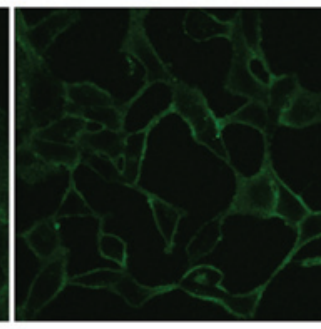

OATP1B1*5

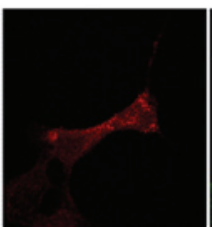

47 (81.04)

$2.31 \quad 11(18.96)$

0.16

1.78

(0.78-4.04)

Figure 1. Correlation of OATP1B1 protein expression with the alleles and genotypes. (A) Imaging of living stable expression OATP1B1 variants shown by confocal laser scanning microscopy, and expression of pcDNA3.1-CT/GFP, pcDNA3.1-GFP-OATP1B1*1a, -OATP1B1*1b, -OATP1B1*5 and -OATP1B1*15 in the HEK293 cells. (B) RT-PCR analysis of mRNA expression of OATP1B1 and GAPDH in HEK293 cells stably expressing the OATP1B1 variants. The OATP1b1 and pcDNA3.1-GFP vectors were used as templates for the positive controls. (C) Double-fluorescence immunocytochemical staining. OATP1B1 was detected by OATP1B1 staining with a Texas Red-tagged secondary antibody, and GFP was detected with a green FITC-tagged secondary antibody. Staining with 4', 6-diamidino-2-phenylindole (DAPI) indicates cell nuclei (blue).

group, but was not significantly different. The *1a and *1b haplotypes $\left(* 1 \mathrm{a} / /^{*} 1 \mathrm{~b},{ }^{*} 1 \mathrm{a} /{ }^{*} 1 \mathrm{a},{ }^{*} 1 \mathrm{~b} /{ }^{*} 1 \mathrm{~b}\right)$ were compared to at least one *15 haplotype $\left({ }^{*} 1 \mathrm{~b} /{ }^{*} 15,{ }^{*} 1 \mathrm{a} / /^{*} 15,{ }^{*} 15 /{ }^{*} 15\right)$, and logistic regression analysis revealed a significant difference between the three patient groups. The patients carrying at least one * 15 haplotype had a significantly increased risk for DILI $(\mathrm{p}=0.03)$, and subjects carrying the * 15 haplotype had a 2.04 -fold significantly elevated risk (95\% CI 1.05-3.96) for DILI. Further analysis of the association between the * 15 haplotype and risk in the subgroup patients revealed that those patients carrying at least one *15 haplotype had a significantly increased risk for
$\mathrm{CS} /$ mixed injury $(\mathrm{p}=0.03, \mathrm{OR}=2.31,95 \% \mathrm{CI} 1.06-5.02)$, but not for HC injury.

Expression of OATP1B1 variants in HEK-293 cells. To determine the effect of the ${ }^{*} 1 \mathrm{a},{ }^{*} 1 \mathrm{~b},{ }^{*} 5$ and ${ }^{*} 15$ haplotypes on the function of OATP1B1, the uptake of TCA by OATP1B1 in the presence of different concentrations of RMP was examined in human HEK293 cells stably expressing OATP1B1 allelic variants (Fig. 1A). The levels of stably expressed OATP1B1 mRNA in the HEK293 cells were analyzed by RT-PCR. The mRNA levels were apparently equal among 


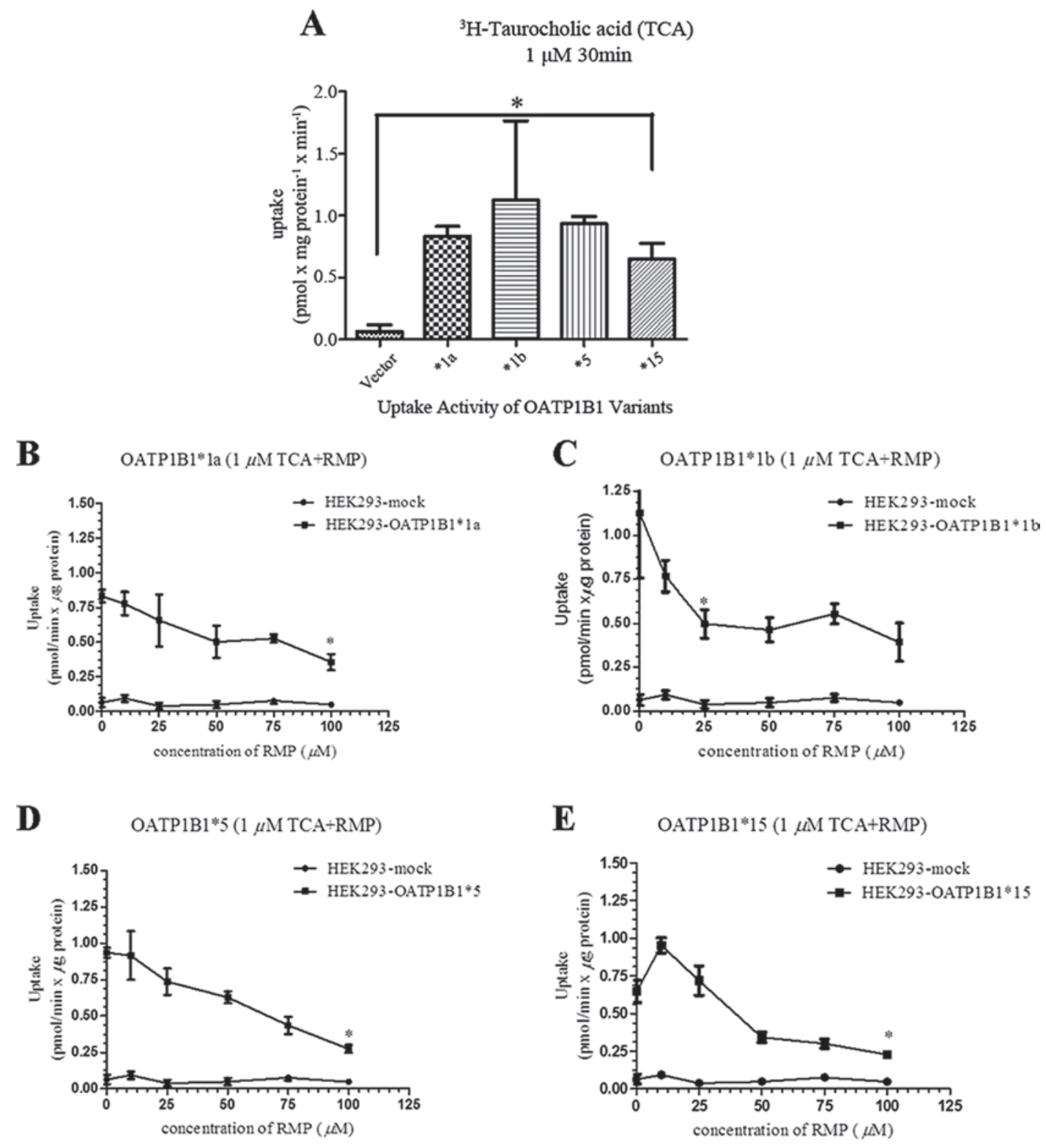

Figure 2. Effects on $\left({ }^{3} \mathrm{H}\right) \mathrm{TCA}$ and rifampin (RMP) uptake in the human embryonic kidney (HEK)293-OATP1B1 variant cells. (A) Uptake of $\left({ }^{3} \mathrm{H}\right) \mathrm{TCA}$ in HEK293 cells (mock cells) and stably expressing OATP1B1 variant cells. All experiments were performed at least twice with triplicate samples and are depicted as the means \pm SE. For each cell transfected with OATP1B1-GFP, asterisks indicate significant differences $(p<0.05)$ from the mock cells. (B-E) Inhibition of the transport of $\left({ }^{3} \mathrm{H}\right)$ TCA by RMP. Various concentrations of RMP were added to the HEK293 cells stably expressing OATP1B1 variants. Data are the means \pm SD of quadruplicate determinations.

the four OATP1B1 variants (Fig. 2B). The cellular locations of each of the OATP1B1 variants were then investigated by immunocytochemical staining. Double-fluorescence and confocal microscopy revealed that GFP-fused OATP1B1 was expressed at the peripheral plasma membrane of the HEK cells (Fig. 1C).

Functional analysis of the OATPIBI variants in vitro. The calculation of the uptake of TCA $(1 \mu \mathrm{M})$ is shown in Fig. 2A. The $\left({ }^{3} \mathrm{H}\right)$ TCA uptake activity of the OATP1B1*1a and other variants differed. There was a significant difference between the HEK 293 cells stably expressing the OATP1B1 variants and the mock cells; the uptake of $\left({ }^{3} \mathrm{H}\right) \mathrm{TCA}$ into the HEK293OATP variant cells was 6-10 times higher than that into the mock cells. In $10 \mathrm{~min}$, the uptake of $\left({ }^{3} \mathrm{H}\right) \mathrm{TCA}$ in OATP1B1*1a,
* $1 \mathrm{~b},{ }^{*} 5$ and *15 averaged $0.834411,1.12441,0.935003$ and 0.647227 pmol x mg protein ${ }^{-1} \times \mathrm{min}^{-1}$, respectively. The uptake of bile acid was reduced in the presence of the * 5 and * 15 haplotypes (both containing the 521C allele) compared to the * $1 \mathrm{~b}$ haplotype (16.9-42.8\%). Next, the inhibitory effect of RMP on the uptake of $\left({ }^{3} \mathrm{H}\right)$ TCA was examined (Fig. 2B-E). The OATP1B1-mediated uptake of $\left({ }^{3} \mathrm{H}\right) \mathrm{TCA}$ was significantly inhibited by RMP. The inhibition of TCA uptake by $100 \mu \mathrm{M}$ RMP was 56, 63, 69 and $68 \%$ in the OATP1B1 ${ }^{*} 1 \mathrm{a},{ }^{*} 1 \mathrm{~b},{ }^{*} 5$ and "15 haplotypes, respectively. The addition of $20 \mu \mathrm{M}$ RMP led to a decrease by ${ }^{*} 1 \mathrm{~b}$ that was more noticeable than that by OATP1B1 ${ }^{*} 1 \mathrm{a},{ }^{*} 5$ and ${ }^{*} 15$. The addition of $100 \mu \mathrm{M}$ RMP resulted in a decreased uptake rate by the * 15 and " 5 variants (both containing the 521C allele) that was greater than that by the ${ }^{*} 1 \mathrm{a}$ and ${ }^{*} 1 \mathrm{~b}$ variants. 


\section{Discussion}

In this study, a systematic screen of all exons and relevant intron-exon boundaries of SLCO1B1 revealed 24 SNPs. Two SNPs that caused amino acid changes in a Chinese population were detected. Based on these two SNPs, four haplotypes, *1a, * $1 \mathrm{~b},{ }^{*} 5$ and ${ }^{*} 15$, in a Chinese population were constructed. The *15 haplotype showed a significant association with the susceptibility to RMP-induced liver injury. The disease-susceptible *15 haplotype had a higher rate of CS/mixed injury. The functional activities of the OATP1B1 variants were further evaluated using a HEK293-OATP1B1 cell system. This in vitro study showed that the 15 haplotype was a functional locus; the uptake activity of TCA was lower than that observed with the other haplotypes and was also significantly inhibited by RMP. Taken together, these results strongly suggest that the OATP1B1 ${ }^{*} 15$ haplotype has a significant association with RMP-induced liver injury. The *15 haplotype may also have a relationship with $\mathrm{CS} /$ mixed type injury.

The two SNPs, 388A>G (Asn130Asp) and 521T>C (Val174Ala), are located within the $S L C O 1 B 1$ gene exons, and their associated amino acids are found at the outer loop between TM3 and TM4 of OATP1B1. No other SNPs that could result in missense mutations were identified. In this study, the association between the four haplotypes constructed with the $388 \mathrm{~A}>\mathrm{G}$ and 521T $>\mathrm{C}$ SNPs and DILI risk were evaluated. The allele frequencies of $388 \mathrm{~A}>\mathrm{G}$ were not significantly different between the three groups, while the allele frequencies of $521 \mathrm{~T}>\mathrm{C}$ were significantly different when the $\mathrm{CS} /$ mixed injury and $\mathrm{HC}$ injury groups were compared to the non-DILI group. The 521T>C SNP has previously been reported to be associated with altered pharmacokinetics of several drugs, including pravastatin, atorvastatin and repaglinide (21-23). The incidence of variations in OATP1B1 is known to be dependent on the racial population. The * $1 \mathrm{~b}$ haplotype is the predominant haplotype in the Chinese population, which is similar to what is observed in the East Asian and sub-Saharan African populations (24). The * 5 and * 15 haplotypes (with frequencies of 0.72 and $9.52 \%$, respectively), which confer decreased OATP1B1 activity, had higher frequencies than those of East Asian populations. Therefore, the impaired OATP1B1 transport activity possibly contributes to the different frequency of RMP-induced liver injury in different populations by the differing frequencies of OATP1B1 variations. Additionally, RMP treatment has been reported to increase serum bile concentrations in patients with the OATP1B1 * 15 haplotype (25), and the OATP1B1 * $3,{ }^{*} 5,{ }^{*} 6$ and ${ }^{*} 13$ variants were reported to reduce the transport of RMP in vitro.

The susceptibility to DILI is influenced by an inter-play between many factors, including increased age, gender (women), concurrent drugs, co-morbidity, transporters and genetics. To the best of our knowledge, this is the first study regarding the association of transport protein SNPs with the risk of RMP-induced liver injury. Among many transport proteins, OATP1B1 plays a significant role in bile salt uptake in the enterohepatic circulation of bile salts (26), and is also a key transporter for RMP. Thus, the functional analyses were preferentially focused on the inhibitory effects of RMP on bile acid transport across HEK293 cells expressing different OATP1B1 variants. TCA was selected to be the substrate for OATP1B1, and the four haplotypes displayed a significant reduction in TCA transport activity. Furthermore, the reduction in the presence of RMP by OATP1B1*5 and *15 (both containing the 521C allele) in the HEK293-OATP1B1 models was greater than that resulting from the ${ }^{*} 1 \mathrm{~b}$ haplotype. The results from other studies also suggest that OATP1B1 is inhibited by RMP in vitro. For example, the reported inhibitory effects on OATP1B1-mediated taurocholate uptake in the presence of RMP were from 58 to $100 \%$, and the decrease of affinity in the mutations also showed a significant reduction of RMP uptake $(12,27)$. Furthermore, several studies have shown that the higher the concentration of RMP, the higher the associated inhibition. For example, $10 \mu \mathrm{M}$ RMP inhibited OATP1B1mediated Sulfobromophthalein (BSP) uptake in vitro by $69 \%$, while $100 \mu \mathrm{M}$ RMP almost completely abolished BSP uptake (24). In our study, the higher the concentration of RMP, the greater the reduction of uptake inhibition.

There were also some limitations in our study. For example, some of our investigated patients were coadministered isoniazid and pyrazinamide with RMP, and thus any confounding effects of isoniazid and pyrazinamide could not be fully eliminated in this study. Nonetheless, the present results indicate that OATP1B1 variants may be involved in the pathogenesis of RMP-induced liver injury and provide a new potential risk factor for patients with this disease.

In conclusion, our genetic epidemiological investigation and functional experimental data suggest that the OATP1B1 *15 haplotype underlies at least some of the phenotypic variations observed in individual susceptibility to RMP-induced liver injury in a Chinese population. Moreover, the *15 haplotype possibly plays an important role in the mechanism by which OATP1B1 transports its substrate.

\section{Acknowledgements}

The authors thank Dr Akira Tsuji (Kanazawa University, Japan) for his kind donation of pcDNA-SLCO1B1. This study was supported by the Chinese National Natural Science Foundation 30900678, 30440015, and the State Key Project for Infectious Diseases (2012ZX10002007-002-005).

\section{References}

1. Prince MI, Burt AD and Jones DE: Hepatitis and liver dysfunction with rifampicin therapy for pruritus in primary biliary cirrhosis. Gut 50: 436-439, 2002.

2. Covic A, Goldsmith DJ, Segall L, Stoicescu C, Lungu S, Volovat C and Covic M: Rifampicin-induced acute renal failure: a series of 60 patients. Nephrol Dial Transplant 13: 924-929, 1998.

3. Wu RQ, Cui XX, Dong WF, Zhou M, Simms HH and Wang P: Suppression of hepatocyte CYP1A2 expression by Kupffer cells via AhR pathway: The central role of proinflammatory cytokines. Int J Mol Med 18: 339-346, 2006.

4. Poole G, Stradling P and Worlledge S: Potentially serious side effects of high-dose twice-weekly rifampicin. Br Med J 3: 343-347, 1971.

5. Chalasani $\mathrm{N}$ and Bjornsson E: Risk factors for idiosyncratic drug-induced liver injury. Gastroenterology 138: 2246-2259, 2010.

6. Fountain FF, Tolley EA, Jacobs AR and Self TH: Rifampin hepatotoxicity associated with treatment of latent tuberculosis infection. Am J Med Sci 337: 317-320, 2009.

7. McCarty MF: 'Iatrogenic Gilbert syndrome' - a strategy for reducing vascular and cancer risk by increasing plasma unconjugated bilirubin. Med Hypotheses 69: 974-994, 2007. 
8. Oelberg DG and Lester R: Cellular mechanisms of cholestasis. Annu Rev Med 37: 297-317, 1986.

9. Weiner M, Peloquin C, Burman W, Luo CC, Engle M, Prihoda TJ, Mac Kenzie WR, Bliven-Sizemore E, Johnson JL and Vernon A: Effects of tuberculosis, race, and human gene SLCO1B1 polymorphisms on rifampin concentrations. Antimicrob Agents Chemother 54: 4192-4200, 2010.

10. Cui Y, Konig J, Leier I, Buchholz U and Keppler D: Hepatic uptake of bilirubin and its conjugates by the human organic anion transporter SLC21A6. J Biol Chem 276: 9626-9630, 2001

11. Li M and Zhang YZ: Modulation of gene expression in cholesterollowering effect of electroacupuncture at Fenglong acupoint (ST40): A cDNA microarray study. Int J Mol Med 19: 617-629, 2007.

12. Vavricka SR, Van Montfoort J, Ha HR, Meier PJ and Fattinger K Interactions of rifamycin SV and rifampicin with organic anion uptake systems of human liver. Hepatology 36: 164-172, 2002.

13. Tirona RG, Leake BF, Wolkoff AW and Kim RB: Human organic anion transporting polypeptide-C (SLC21A6) is a major determinant of rifampin-mediated pregnane $\mathrm{X}$ receptor activation. J Pharmacol Exp Ther 304: 223-228, 2003.

14. Lu WJ, Tamai I, Nezu J, Lai ML and Huang JD: Organic anion transporting polypeptide-C mediates arsenic uptake in HEK-293 cells. J Biomed Sci 13: 525-533, 2006.

15. Niemi M, Pasanen MK and Neuvonen PJ: Organic anion transporting polypeptide 1B1: a genetically polymorphic transporter of major importance for hepatic drug uptake. Pharmacol Rev 63: 157-181, 2011.

16. Danan $G$ and Benichou C: Causality assessment of adverse reactions to drugs - I. A novel method based on the conclusions of international consensus meetings: application to drug-induced liver injuries. J Clin Epidemiol 46: 1323-1330, 1993.

17. Benichou C: Criteria of drug-induced liver disorders. Report of an international consensus meeting. J Hepatol 11: 272-276, 1990.

18. Kruglyak L and Nickerson DA: Variation is the spice of life. Nat Genet 27: 234-236, 2001

19. Kameyama Y, Yamashita K, Kobayashi K, Hosokawa M and Chiba K: Functional characterization of SLCO1B1 (OATP-C) variants, SLCO1B1*5, SLCO1B1*15 and SLCO1B1*15+C1007G, by using transient expression systems of HeLa and HEK293 cells. Pharmacogenet Genomics 15: 513-522, 2005.
20. Byrne JA, Strautnieks SS, Mieli-Vergani G, Higgins CF, Linton KJ and Thompson RJ: The human bile salt export pump: characterization of substrate specificity and identification of inhibitors. Gastroenterology 123: 1649-1658, 2002.

21. Mwinyi J, Johne A, Bauer S, Roots I and Gerloff T: Evidence for inverse effects of OATP-C (SLC21A6) 5 and 1b haplotypes on pravastatin kinetics. Clin Pharmacol Ther 75: 415-421, 2004.

22. Lau YY, Okochi H, Huang Y and Benet LZ: Multiple transporters affect the disposition of atorvastatin and its two active hydroxy metabolites: application of in vitro and ex situ systems. J Pharmacol Exp Ther 316: 762-771, 2006.

23. Niemi M, Backman JT, Kajosaari LI, Leathart JB, Neuvonen M, Daly AK, Eichelbaum M, Kivisto KT and Neuvonen PJ: Polymorphic organic anion transporting polypeptide $1 \mathrm{~B} 1$ is a major determinant of repaglinide pharmacokinetics. Clin Pharmacol Ther 77: 468-478, 2005.

24. Abe T, Kakyo M, Tokui T, Nakagomi R, Nishio T, Nakai D, Nomura H, Unno M, Suzuki M, Naitoh T, et al: Identification of a novel gene family encoding human liver-specific organic anion transporter LST-1. J Biol Chem 274: 17159-17163, 1999.

25. Ieiri I, Suzuki H, Kimura M, Takane H, Nishizato Y, Irie S, Urae A, Kawabata K, Higuchi S, Otsubo K, et al: Influence of common variants in the pharmacokinetic genes (OATP-C, UGT1A1, and MRP2) on serum bilirubin levels in healthy subjects. Hepatol Res 30: 91-95, 2004

26. Jindal C, Kumar S, Choudhari G, Goel H and Mittal B: Organic anion transporter protein (OATP1B1) encoded by SLCO1B1 gene polymorphism $(388 \mathrm{~A}>\mathrm{G})$ and susceptibility in gallstone disease. Indian J Med Res 129: 170-175, 2009.

27. Fattinger K, Cattori V, Hagenbuch B, Meier PJ and Stieger B: Rifamycin SV and rifampicin exhibit differential inhibition of the hepatic rat organic anion transporting polypeptides, Oatp1 and Oatp2. Hepatology 32: 82-86, 2000. 